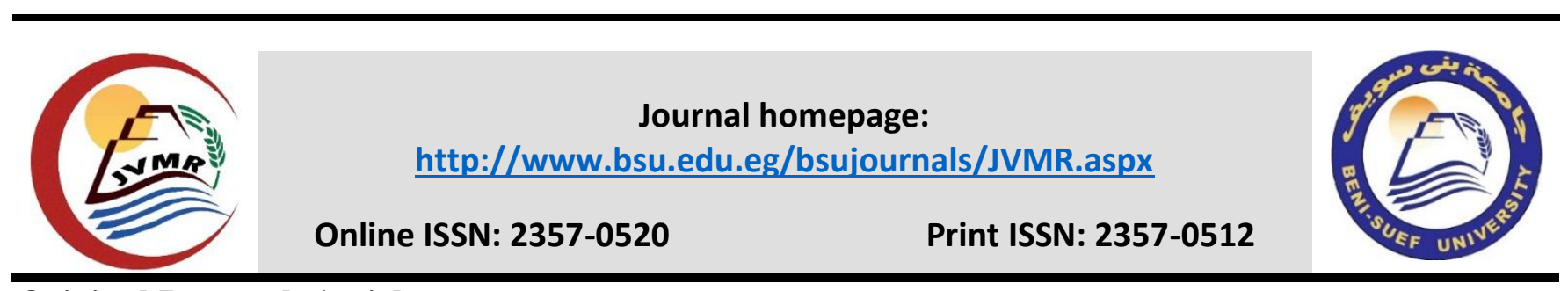

Original Research Article

\title{
Characterization of Avian Influenza H9N2 and Newcastle Disease Virus Isolated from Vaccinated Chickens in Upper Egypt
}

Safaa A. A. Abdel-Latif ${ }^{1 \#}$, Asmaa Atef ${ }^{2 \#}$, Ahmed M. A. Abdel-Aleem ${ }^{3}$, AL-Hussien M. Dahshan ${ }^{4}$, Ahmed Ali ${ }^{4 *}$.

${ }^{1}$ Directorate of Veterinary Medicine in Minia, General Organization of Veterinary Services, Ministry of Agriculture, Egypt

${ }^{2}$ General Administration of Student Housing Facilities, Fayoum University, Egypt.

${ }^{3}$ General Administration of student housing facilities, Beni Suef University, Beni Suef 62511 Egypt.

${ }^{4}$ Poultry Diseases Department, Faculty of Veterinary Medicine, Beni Suef University, Beni Suef 62511 Egypt.

\section{ABSTRACT}

In this study, 50 vaccinated broiler flocks and one layer flock from Beni Suef, Fayoum and Minia Governorates were investigated. Necropsy lesions were suggestive of LPAI-H9N2 or NDV. Samples including tracheal swabs and organs were subjected for viral isolation and molecular characterization. Specific RT-PCR for the F-gene of NDV and the HA gene of the LPAI-H9N2 viruses was used. Virus isolation and primary identification using $\mathrm{HI}$ test revealed 37.5 and $43.3-46.2 \%$ prevalence for LPAI-H9N2 and NDV viruses, respectively. Phylogenetic analysis of partial sequences of the $\mathrm{F}$ gene showed that NDV viruses belong to genotype II and VII-1.1. as indicated by the F0 protein proteolytic cleavage site motifs (aa112-117) of the NDV strains F-gene. The vNDV isolates were 98.7-99.3\% and 96.6-98.9\% identical to each other based on nucleotide and amino acid identities, respectively. Compared to their counterpart isolates; the lentogenic strains shared $98-99.2 \%$ and 96.3-98.1\% nucleotide and amino acid identities to the LaSota reference strain. The LPAI-H9N2 phylogeny of the HA gene showed that the 2 isolates obtained in this study are related to each other and related to recent 2016-2018 Egyptian H9N2 strains. Notably, the 2 strains showed higher identity ( $\geq 99 \%)$ to recent Israeli 2018 isolates with several amino acid changes. The current study revealed widespread of both NDV and LPAI-H9N2 viruses. The vaccine failure and the mismatch between the vaccine and circulating NDV viruses is the most probable cause of current outbreaks. LPAI-H9N2 viruses are divergent from their ancestral viruses in Egypt indicating continuous circulation and vaccine pressure-induced mutations.
ARTICLE INFO Article history:

Received 11/3/2020

Accepted 12/5/2020

Online 13/5/2020

\section{Keywords:}

Broiler Chickens, Egypt, H9N2, LPAI, NDV, Surveillance.

Corresponding author: D. Ahmed Ali. Poultry Diseases Department, Faculty of Veterinary Medicine, Beni Suef University, Beni Suef 62511 Egypt Email: ahmed.ali1@ vet.bsu.edu.eg 


\section{Introduction}

Poultry production constitutes one of the main sources of protein in Egypt. However, respiratory disease challenges have been rising in Egyptian commercial flocks during the last decade (Hassan et al., 2016). Considering the non-pathognomonic characteristics of respiratory diseases, they are frequently misdiagnosed (Peyre et al., 2009). Mixed and secondary infections may interfere with the diagnosis of the primary cause due to the absence of perceiving signs and lesions (KarimiMadab et al., 2010; Samy and Naguib, 2018). Different pathogens such as avian influenza (AI), Newcastle disease (ND), and infectious bronchitis (IB) viruses are involved either in single or multiple respiratory infections (Nili and Asasi, 2002; Hassan et al., 2017; Setta et al., 2018).

Influenza A viruses are negative-sense, single-stranded RNA viruses of the family Orthomyxoviridae with 18 hemagglutinins (HA) and 11 neuraminidase (NA) subtypes. The low pathogenic avian influenza H9N2 (LPAI-H9N2) viruses first recorded in turkeys in Wisconsin in 1966 (Swayne and Brown, 2015) cause mild to severe respiratory signs, but they are mainly associated with a reduction in egg production in breeders and layers (Shehata et al., 2015). Serologically, the virus antibodies were found in poultry between 2009 to 2012 in Egypt (Afifi et al., 2012). However, virus isolation was confirmed in Egypt in December 2010 (ElZoghby et al., 2012).

Most respiratory outbreaks associated with LPAI-H9N2 viruses are reported during winter months (Hassan et al., 2016), however, recent reports showed that the virus is circulating all over the year, especially in the Nile Delta (Nagy et al., 2017). The isolated viruses were genetically related to the viruses circulating in the Middle East region and to those isolated from the migratory birds.
Moreover, the infected flocks were distributed along with the migratory birds' flyways suggesting their introduction via wild migratory birds (Abdelwhab and Abdel-Moneim, 2015).

Newcastle disease (ND) is recently classified according to The International Committee on Taxonomy of Viruses (ICTV) to be a member of family Paramyxoviridae, subfamily Avulavirinae, genus Orthoavulavirus, species Avian orthoavulavirus 1 (Dimitrov et al., 2019). NDV strains are separated into two clades, class I and class II, within a single serotype (Czeglédi et al., 2006). Class I viruses comprise a single avirulent genotype and class II viruses are further divided into 18 genotypes (Afonso et al., 2012; Miller, 2010). Class II strains include most virulent NDVs (Czeglédi et al., 2006), of which genotypes I to IV represent early sub-lineages (Ewies et al., 2017; Mohamed et al., 2011; Radwan et al., 2013). Though intensive NDV vaccination programs applied in Egypt, many outbreaks of NDV by genotype VII 1.1. have been recorded (Ewies et al., 2017; Mohamed et al., 2011; Radwan et al., 2013).

In this study, LPAI H9N2 and NDV viruses were from recent outbreaks in broiler chickens from Upper Egypt isolated and molecularly characterized (Fayoum, Beni Suef, and Minia Governorates).

\section{Material and Methods}

\section{Field samples}

Fifty vaccinated broiler-type and one layer-type flocks from Beni Suef (8 flocks), Fayoum (13 flocks), and Minia (30 flocks) Governorates were included in this study. Investigated flocks suffered from respiratory distress and nervous signs were reported in some flocks (Table 1). Post-mortem lesions were suggestive for either LPAI H9N2 or NDV. Collected samples included tracheal swabs, trachea and lungs, and cecal tonsils. 
Table 1. History of the collected field samples.

\begin{tabular}{|c|c|c|c|c|c|c|c|}
\hline Sample & $\begin{array}{c}\text { Age } \\
\text { (days) }\end{array}$ & $\begin{array}{c}\text { Flock } \\
\text { size }\end{array}$ & $\begin{array}{c}\text { Productio } \\
\text { n type }\end{array}$ & Postmortem lesions & Vaccination program & $\begin{array}{l}\text { Sampling } \\
\text { date }\end{array}$ & \\
\hline AA005 & 27 & 3000 & broiler & CRD, hemorrhage at cecal tonsils & \multirow{3}{*}{$\begin{array}{l}\text { Day7: IBV+ND HB1- Day17: ND } \\
\text { LaSota }\end{array}$} & \multirow{3}{*}{ Dec-2015 } & Fayoum \\
\hline AA012 & 20 & 6500 & broiler & $\begin{array}{l}\text { Bloody intestine- congested } \\
\text { trachea and liver }\end{array}$ & & & Fayoum \\
\hline AA006 & 28 & 7000 & broiler & Hemorrhage at cecal tonsils & & & Fayoum \\
\hline AA007 & 15 & 4000 & layer & $\begin{array}{l}\text { Hemorrhage at tips of the } \\
\text { proventriculus }\end{array}$ & $\begin{array}{l}\text { Day1: IB primer } \\
\text { Day7: Killed ND }\end{array}$ & Mar-2016 & Fayoum \\
\hline AA010 & 26 & $\begin{array}{l}2 \mathrm{sa} 024 \\
000\end{array}$ & layer & $\begin{array}{l}\text { Hemorrhages at tips of the } \\
\text { proventriculus. Severe congestion } \\
\text { in carcass }\end{array}$ & $\begin{array}{l}\text { Day6: IBV+ND HB1- } \\
\text { Day17: ND LaSota }\end{array}$ & \multirow{2}{*}{ May-2016 } & Fayoum \\
\hline AA011 & 13 & 3500 & broiler & CRD. Hemorrhagic intestine & $\begin{array}{l}\text { Day1: IBV+ND HB1- Day7: Killed } \\
\text { ND } \\
\text { Day17: ND LaSota }\end{array}$ & & Fayoum \\
\hline AA009 & 7 & 5000 & broiler & $\begin{array}{l}\text { Liver congestion, minor } \\
\text { hemorrhages at the caecum }\end{array}$ & Day6: IBV+ND HB1 & Dec-2016 & Fayoum \\
\hline SA001 & 27 & 6000 & broiler & $\begin{array}{l}\text { CRD, Ascites, congested heart, } \\
\text { liver, spleen }\end{array}$ & $\begin{array}{l}\text { Day6= IBV+ND HB1- Day9: Killed } \\
\text { H5+ND- Day11: IBD intermediate } \\
\text { plus- Day17: ND LaSota- Day19: IBD } \\
\text { intermediate plus }\end{array}$ & \multirow[t]{2}{*}{ Jan-2017 } & Minia \\
\hline SA002 & 27 & 5000 & broiler & $\begin{array}{l}\text { Congested trachea with mucous } \\
\text { exudate }\end{array}$ & $\begin{array}{l}\text { Day7: Killed H5- Day8: Killed ND } \\
\text { vaccine- Day17: ND LaSota }\end{array}$ & & Minia \\
\hline AA008 & 9 & 1500 & broiler & General Congestion & Day1: IBV+ND HB1 & Feb-2017 & Fayoum \\
\hline SA006 & 25 & 9000 & broiler & CRD, cecal coccidiosis, tracheitis & $\begin{array}{l}\text { Day8: IB and ND Colone30- Day10: } \\
\text { Killed H5+ND-Day12: IBD } \\
\text { intermediate plus-Day16: ND Clone30 }\end{array}$ & \multirow[t]{2}{*}{ Mar-2017 } & Minia \\
\hline SA007 & 31 & 2500 & broiler & CCRD, air saculitis, bronchitis & $\begin{array}{l}\text { Day1: IBV+ND HB1- Day16: ND } \\
\text { Clone } 30\end{array}$ & & Minia \\
\hline SA008 & 39 & 2000 & broiler & $\begin{array}{l}\text { CRD, clostridial enteritis, cecal } \\
\text { core }\end{array}$ & $\begin{array}{l}\text { Day6: IBV+ND HB1- Day19: ND } \\
\text { LaSota }\end{array}$ & Apr-2017 & Minia \\
\hline
\end{tabular}




\begin{tabular}{|c|c|c|c|c|c|c|c|}
\hline SA011 & 31 & 3000 & broiler & CRD, enteritis, ascites & $\begin{array}{l}\text { Day7: ND Colone30+ IB MA5- } \\
\text { Day10: KilledH5+ND, Day13: IBD } \\
\text { intermediate plus }\end{array}$ & & Minia \\
\hline SA012 & 30 & 3000 & broiler & CRD, hydropericardium, ascites, & Day6: IBV+ND HB1- Day11: IBD & & Minia \\
\hline SA015 & 29 & 2000 & broiler & air saculitis & intermediate plus-Day18: ND LaSota & & Minia \\
\hline SA017 & 32 & 5000 & broiler & $\begin{array}{l}\text { Greenish diarrhea, air saculitis, } \\
\text { pericarditis }\end{array}$ & $\begin{array}{l}\text { Day1: IBV- Day7: Killed H5N2 and } \\
\text { ND- Day17: ND LaSota }\end{array}$ & & Minia \\
\hline AA004 & 11 & 6000 & broiler & $\begin{array}{l}\text { Hemorrhages at tips of the } \\
\text { proventriculus }\end{array}$ & $\begin{array}{l}\text { Day6: IBV+ND HB1- Day6: Killed } \\
\text { ND vaccine }\end{array}$ & & Fayoum \\
\hline SA009 & 27 & 2000 & broiler & CRD, air saculitis, bronchitis & \multirow{2}{*}{$\begin{array}{l}\text { Day1: IBV- Day7: ND HB1-Day12: } \\
\text { IBD intermediate plus }\end{array}$} & \multirow{11}{*}{ May-2017 } & Minia \\
\hline SA010 & 29 & 4500 & broiler & $\begin{array}{l}\text { CRD, air saculitis, bronchitis, } \\
\text { greenish diarrhea }\end{array}$ & & & Minia \\
\hline SA013 & 30 & 5000 & broiler & CRD, air saculitis, bronchitis & $\begin{array}{l}\text { Day7: ND HB1 and IBD } \\
\text { (VAXXITEC)- Day10: Killed } \\
\text { H5+ND- Day17: ND Clone } 30\end{array}$ & & Minia \\
\hline SA014 & 29 & 6000 & broiler & $\begin{array}{l}\text { CRD, air saculitis, bronchitis, } \\
\text { greenish diarrhea, cecal } \\
\text { coccidiosis, general septicemia }\end{array}$ & $\begin{array}{l}\text { Day7: ND HB1- Day14d: IBD } \\
\text { intermediate plus- Day18: ND } \\
\text { Clone30 }\end{array}$ & & Minia \\
\hline SA016 & 26 & 4000 & broiler & $\begin{array}{l}\text { Air saculitis, fibrinous } \\
\text { pericarditis, hemorrhagic } \\
\text { bronchitis }\end{array}$ & $\begin{array}{l}\text { Day7: ND HB1- Day8: Killed ND- } \\
\text { Day13: IBD intermediate plus }\end{array}$ & & Minia \\
\hline SA024 & 29 & 1000 & broiler & $\begin{array}{l}\text { Whitish diarrhea, mild clostridial } \\
\text { enteritis, bronchitis }\end{array}$ & $\begin{array}{l}\text { Day6: ND HB1- Day12: IBD } \\
\text { intermediate plus }\end{array}$ & & Minia \\
\hline SA018 & 22 & 4000 & broiler & $\begin{array}{l}\text { Air saculitis, greenish diarrhea, } \\
\text { septicemia }\end{array}$ & Not available & & Minia \\
\hline SA019 & 32 & 1200 & Sasso & pericarditis, cecal core, enteritis & Not available & & Minia \\
\hline SA030 & 32 & 4000 & broiler & CRD, greenish diarrhea, ascites & $\begin{array}{l}\text { Day8: ND HB1- Day12: IBD } \\
\text { intermediate plus- Day16: ND } \\
\text { Clone30 }\end{array}$ & & Minia \\
\hline SA027 & 24 & 5000 & broiler & $\begin{array}{l}\text { CRD, IBD postvaccinal reaction, } \\
\text { air saculitis }\end{array}$ & $\begin{array}{l}\text { Day6: ND HB1- Day8: Killed } \\
\text { H5+ND- Day12: IBD intermediate } \\
\text { plus }\end{array}$ & & Minia \\
\hline SA021 & 25 & 40000 & broiler & CRD, greenish diarrhea & $\begin{array}{l}\text { Day8: ND HB1- Day12: IBD } \\
\text { intermediate plus- Day17: ND }\end{array}$ & & Minia \\
\hline
\end{tabular}


JOURNAL OF VETERINARY MEDICAL RESEARCH 2020, 27 (1): 90-108

\begin{tabular}{|c|c|c|c|c|c|c|c|}
\hline & & & & & Clone30 & & \\
\hline SA022 & 30 & 3500 & broiler & $\begin{array}{l}\text { Bronchitis, greenish diarrhea, air } \\
\text { saculitis }\end{array}$ & $\begin{array}{l}\text { Day8: ND HB1- Day12: IBD } \\
\text { intermediate plus- Day18: ND LaSota }\end{array}$ & & Minia \\
\hline SA023 & 18 & 2500 & broiler & $\begin{array}{l}\text { CRD, ascites, nephrosis, greenish } \\
\text { diarrhea }\end{array}$ & $\begin{array}{l}\text { Day8: ND HB1- Day13: IBD } \\
\text { intermediate plus }\end{array}$ & & Minia \\
\hline SA026 & 35 & 1700 & broiler & CRD, greenish diarrhea, enteritis & Not available & & Minia \\
\hline SA028 & 45 & 5000 & broiler & $\begin{array}{l}\text { CRD, greenish diarrhea, cecal } \\
\text { coccidiosis }\end{array}$ & $\begin{array}{l}\text { Day6: ND HB1- Day7: Killed } \\
\text { H5+ND- Day10: IBD intermediate } \\
\text { plus }\end{array}$ & & Minia \\
\hline SA025 & 30 & 4000 & broiler & CRD, nephrosis, air saculitis & $\begin{array}{l}\text { Day7: ND HB1- Day11: IBD } \\
\text { intermediate plus- Day15: ND LaSota }\end{array}$ & \multirow{2}{*}{ Jun-2017 } & Minia \\
\hline SA035 & 21 & 2750 & broiler & $\begin{array}{l}\text { perihepatitis, air saculitis, } \\
\text { bronchitis }\end{array}$ & Not available & & Minia \\
\hline SA003 & 35 & 2000 & Sasso & CRD, greenish diarrhea & Day8: ND HB1 - Day18: ND Clone30 & \multirow{3}{*}{ Oct-2017 } & Minia \\
\hline SA004 & 32 & 5000 & broiler & $\begin{array}{l}\text { CCRD, mild clostridia, nephrosis, } \\
\text { mycotoxicosis }\end{array}$ & \multirow{2}{*}{$\begin{array}{l}\text { Day8: Killed H5N2+ND- Day10: } \\
\text { IBV+ND HB1- Day12: IBD } \\
\text { intermediate plus- Day18: ND LaSota }\end{array}$} & & Minia \\
\hline SA005 & 32 & 8000 & broiler & CRD, cecal coccidiosis & & & Minia \\
\hline SA020 & 32 & 6500 & broiler & $\begin{array}{l}\text { CRD, nephrosis, greenish } \\
\text { diarrhea }\end{array}$ & $\begin{array}{l}\text { Day7: ND HB1-Day8: } \\
\text { KilledH5N2+ND-Day12: IBD } \\
\text { intermediate plus }\end{array}$ & Dec-2017 & Minia \\
\hline AA001 & 10 & 2000 & broiler & hemorrhages at cecal tonsils & $\begin{array}{l}\text { Day1: IBV+ND HB1- Day7: Killed } \\
\text { ND }\end{array}$ & Jan-2018 & Fayoum \\
\hline AM001 & 34 & 13000 & broiler & $\begin{array}{l}\text { Tracheal cast, severe congestion } \\
\text { in trachea and kidney }\end{array}$ & $\begin{array}{l}\text { Day1: IBV+ ND Clone30- Day7: } \\
\text { Killed H9+ND- Day13: IBD } \\
\text { intermediate plus }\end{array}$ & \multirow{6}{*}{ Mar-2018 } & $\begin{array}{l}\text { Beni- } \\
\text { Suef }\end{array}$ \\
\hline AA002 & 18 & 650 & broiler & $\begin{array}{l}\text { Hemorrhages at tips of the } \\
\text { proventriculus }\end{array}$ & Day7: ND LaSota- Day17: Killed ND & & Fayoum \\
\hline AA003 & 9 & 2500 & broiler & Tracheal cast, liver congestion & $\begin{array}{l}\text { Day7: IBV+ ND HB1-Day7: Killed } \\
\text { ND }\end{array}$ & & Fayoum \\
\hline AM002 & 33 & 4000 & broiler & \multirow{2}{*}{$\begin{array}{l}\text { Severe congestion in trachea and } \\
\text { kidney, mild CRD }\end{array}$} & \multirow{2}{*}{$\begin{array}{l}\text { Day1: IB and ND Colone30- Day7: } \\
\text { Killed H9+ND-Day13: IBD } \\
\text { intermediate plus }\end{array}$} & & Fayoum \\
\hline AM003 & 33 & 5000 & broiler & & & & $\begin{array}{l}\text { Beni } \\
\text { Suef }\end{array}$ \\
\hline AM004 & 23 & 3500 & broiler & $\begin{array}{l}\text { Tracheal cast, congested trachea, } \\
\text { nephritis, hemorrhage in thigh }\end{array}$ & $\begin{array}{l}\text { Day5: Killed ND- Day7: IBV+ ND } \\
\text { HB1- Day13: IBD intermediate plus- }\end{array}$ & & $\begin{array}{l}\text { Beni } \\
\text { Suef }\end{array}$ \\
\hline
\end{tabular}


Abdel-Latif et al. (2020)

\begin{tabular}{|c|c|c|c|c|c|c|c|}
\hline & & & & muscle & Day19: ND LaSota & & \\
\hline AM007 & 30 & 5500 & broiler & $\begin{array}{l}\text { Severe congestion in trachea, } \\
\text { nephritis, dehydration, severe } \\
\text { congestion in kidney }\end{array}$ & $\begin{array}{l}\text { Day4: H9+IBD Galimune- Day9: } \\
\text { KilledH5+ ND Colone30+ IBV MA5- } \\
\text { Day12: IBD intermediate plus- } \\
\text { Day18: ND LaSota }\end{array}$ & \multirow{5}{*}{ Apr-2018 } & $\begin{array}{l}\text { Beni } \\
\text { Suef }\end{array}$ \\
\hline AM010 & 27 & 3000 & broiler & $\begin{array}{l}\text { Congested trachea and liver, } \\
\text { ascites, bloody diarrhea }\end{array}$ & $\begin{array}{l}\text { Day6: IBV+ND Clone30- Day9: } \\
\text { Killed H9+ND-Day13 IBD } \\
\text { intermediate plus }\end{array}$ & & $\begin{array}{l}\text { Beni } \\
\text { Suef }\end{array}$ \\
\hline AM008 & 32 & 2000 & broiler & $\begin{array}{l}\text { Severe congestion in trachea, } \\
\text { nephritis, severe congestion in } \\
\text { kidney }\end{array}$ & $\begin{array}{l}\text { Day5: IB+Clone30- Day9: Killed H9- } \\
\text { Day13: IBD intermediate plus-Day16: } \\
\text { IB MA5+Clone30 }\end{array}$ & & $\begin{array}{l}\text { Beni } \\
\text { Suef }\end{array}$ \\
\hline AM005 & 30 & 7000 & broiler & \multirow{2}{*}{$\begin{array}{l}\text { Severe congestion in trachea, } \\
\text { dehydration, severe congestion in } \\
\text { the kidney, hemorrhage in thigh } \\
\text { muscle }\end{array}$} & $\begin{array}{l}\text { Day7: IB+Clone30 and Killed H5- } \\
\text { Day9: Killed H9- Day13: IBD } \\
\text { intermediate plus-Day16: IB } \\
\text { MA5+Clone30 }\end{array}$ & & $\begin{array}{l}\text { Beni } \\
\text { Suef }\end{array}$ \\
\hline AM009 & 32 & 2500 & broiler & & $\begin{array}{l}\text { Day7: IB+ ND Hitchner IB- Day12: } \\
\text { IBD intermediate plus-Day19: IB+ } \\
\text { ND Hitchner }\end{array}$ & & $\begin{array}{l}\text { Beni } \\
\text { Suef }\end{array}$ \\
\hline
\end{tabular}


Sample Processing, Virus Detection, and Virus Isolation

Tissue and/or swab samples were pooled and processed in sterile phosphate buffer saline $\mathrm{pH}$ 7.0-7.4 containing gentamycin $(50 \mu \mathrm{g} / \mathrm{ml})$ (Ewies et al., 2017). Supernatants were inoculated into the allantoic sac of 9-day-old specific pathogen free embryonated chicken eggs (SPF-ECE) (Fouchier et al., 2000). Inoculated eggs were incubated at $37^{\circ} \mathrm{C}$ for 72 hours and candled daily for embryo viability. The collected allantoic fluids from inoculated eggs were tested for hemagglutination using $1 \%$ washed chicken RBCs (Swayne and Brown, 2015).

\section{RT-PCR and Gene Sequencing}

The viral RNA was extracted from harvested allantoic fluids by Viral Gene-Spin ${ }^{\mathrm{TM}}$ viral DNA/RNA extraction kit (iNtRON Biotechnology Inc., China) according to the manufacturer's instructions. RT-PCR was used for the detection of the F-gene of vNDV and the hemagglutinin (HA) gene of the LPAI H9N2 viruses using Specific primers (Table 2). A single step RRT-PCR assays using TOPscript ${ }^{\mathrm{TM}}$ One-Step RT-PCR Kit (Enzyomics Inc., China) was used according to the manufacturer's instructions. The final reaction volume was 20 $\mu \mathrm{L}$, including $5 \mu \mathrm{L}$ RNA template, $5 \mu \mathrm{L}$ TOPscript $^{\mathrm{TM}}$ One-Step RT-PCR Kit, $1 \mu \mathrm{L}$ of each forward and reverse primers $(20 \mathrm{pmol})$, and $8 \mu \mathrm{L}$ RNase-free water. Thermal cycling RT-PCR conditions included a reverse transcription at $50^{\circ} \mathrm{C}$ for $30 \mathrm{~min}$, then an inactivation of reverse transcription enzyme and initial denaturation at $95^{\circ} \mathrm{C}$ for $10 \mathrm{~min}$, followed by 40 cycles at $95^{\circ} \mathrm{C}$ for $30 \mathrm{sec}$., $45 \mathrm{sec}$ at $47-$ $51^{\circ} \mathrm{C}$ (Table 2), and $2 \mathrm{~min}$ at $72^{\circ} \mathrm{C}$. The addition final extension was performed at $72^{\circ} \mathrm{C}$ for 10 min.

Table 2. Oligonucleotide primers for amplification of the NDV F gene and LPAI H9N2 HA gene.

\begin{tabular}{|c|c|c|c|c|c|}
\hline Virus & & Primer & $\begin{array}{l}\text { Annealing } \\
\text { temperature } \\
\left({ }^{\circ} \mathbf{C}\right)\end{array}$ & $\begin{array}{l}\text { Size } \\
\text { (bp) }\end{array}$ & Reference \\
\hline \multirow{2}{*}{\multicolumn{2}{|c|}{$\begin{array}{l}\text { Virulent } \\
\text { NDV }\end{array}$}} & F-5’ ATGGGCTCCAAACCTTCTA-‘3 & \multirow{2}{*}{50} & \multirow{2}{*}{1600} & \multirow{2}{*}{$\begin{array}{l}\text { (Nagy et } \\
\text { al., 2020) }\end{array}$} \\
\hline & & R-5'GGAAACCTTCGTTCCTCAT-‘3 & & & \\
\hline \multirow{4}{*}{$\begin{array}{l}\text { LPAI } \\
\text { h9N2 }\end{array}$} & \multirow{2}{*}{ Seg1 } & $\begin{array}{l}\text { F-5' TATTCGTCTCAGGGAGCAAAAGCAGG- } \\
\text { ‘3 }\end{array}$ & \multirow{2}{*}{47} & \multirow{2}{*}{913} & $\begin{array}{l}\text { (Hoffmann } \\
\text { et al., 2001) }\end{array}$ \\
\hline & & $\begin{array}{l}\text { R-5'ATTACGTCTC- } \\
\text { TGTGGAAAGGTAATGTACTG-`3 }\end{array}$ & & & $\begin{array}{l}\text { (Shany, } \\
2015)\end{array}$ \\
\hline & \multirow[b]{2}{*}{ Seg } & $\begin{array}{l}\text { F-5’ ATTACGTCTC- } \\
\text { TCCACAATATCAGTAAATAT-`3 }\end{array}$ & \multirow[b]{2}{*}{51} & \multirow[b]{2}{*}{754} & $\begin{array}{l}\text { (Shany, } \\
\text { 2015) }\end{array}$ \\
\hline & & $\begin{array}{c}\text { R- } \\
\text { 5'ATATCGTCTCGTATTAGTAGAAACAAGG- }\end{array}$ & & & $\begin{array}{l}\text { (Hoffmann } \\
\text { et al., 2001) }\end{array}$ \\
\hline
\end{tabular}


The RT-PCR products of the target bands were purified after gel electrophoresed using the MEGAquick-spin ${ }^{\mathrm{TM}}$ Plus total fragment DNA purification kit (iNtRON Biotechnology Inc., China) according to the manufacturer instructions and the DNA was shipped for sequencing at Macrogen, Korea. Sequence comparisons and phylogenetic relationships

\section{Results}

\section{Virus Isolation}

The mortality in infected chicken ranged between $15-20 \%$ and necropsy revealed tracheitis with petechial hemorrhage on were determined with the MEGA X software the Clustal W alignment algorithm (Kumar et al., 2018). Nucleotide and deduced amino acid sequence analysis of $\mathrm{F}$ gene of NDV and HA gene of LPAI-H9N2 were conducted in comparison with vaccinal and virulent Egyptian NDV isolates using Geneious ${ }^{\circledR}$ 7.1.3, Copyright (C) 2005-2014 Biomatters Inc.

Table 3. Isolation rates of NDV and LPAI-H9N2 viruses from the flocks under investigation

\begin{tabular}{rcccc} 
Location & & Beni Suef & Fayoum & Minia \\
\hline No of samples & & 8 & 13 & 30 \\
\hline \multirow{3}{*}{ RT-PCR Result } & NDV & $3(37.5 \%)$ & $6(46.1 \%)$ & $13(43.3 \%)$ \\
\cline { 2 - 5 } & H9N2 & $0(0 \%)$ & $1(7.7 \%)$ & $2(6.7 \%)$ \\
\cline { 2 - 5 } & Neg & $5(62.5 \%)$ & $6(46.1 \%)$ & $15(50.0 \%)$ \\
\hline $\begin{array}{c}\text { Isolation (out of } \\
\text { positive samples) }\end{array}$ & NDV & $2(66.7 \%)$ & $1(16.7 \%)$ & $4(30.8 \%)$ \\
\cline { 2 - 5 } & H9N2 & $0(0 \%)$ & $1(100 \%)$ & $1(50.0 \%)$ \\
\hline
\end{tabular}

\section{Phylogeny and Genetic Analysis of the vNDV strains sequences}

The isolated NDV belong to wither genotype II or genotype VII 1.1. (Figure 1). Both genotypes retained the previously characterized amino acid sequences of the $\mathrm{F} 0$ protein cleavage site motifs $\left({ }^{112}\right.$ GRQGRL ${ }^{117}$ motif and ${ }^{112}$ RRQKRF $^{117}$ motif for lentogenic and velogenic genotype, respectively). Few amino acid substitutions were observed (Figure 2). The vNDV isolates in this study were $98.7-99.3 \%$ and $96.6-98.9 \%$ identical to each other based on nucleotide and amino acid identities, respectively. proventriculus. Results of virus isolation and primary identification using $\mathrm{HI}$ test are summarized in table 3. 


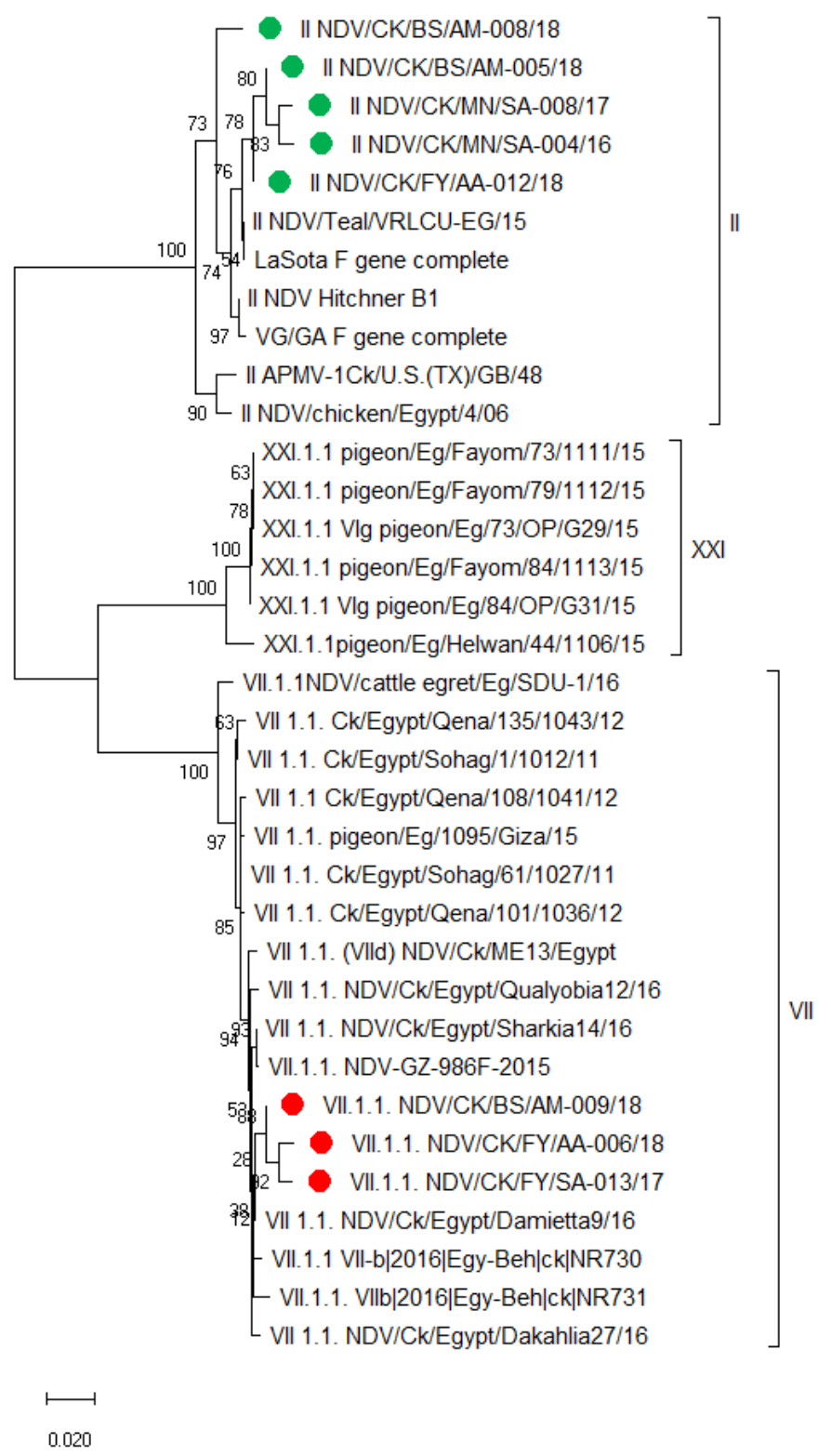

Fig. 1. Phylogenetic analysis of the partial F-gene sequence of isolated lentogenic NDV (green dots) and vNDV strains (red dots). Abbreviations: (EG, Egypt; CK, chicken). Representative strains from different genotypes were included. Phylogenetic relationships through a bootstrap trial of 1000 were determined with the MEGA version 6 using the Clustal W alignment algorithm and neighbor-joining method for tree construction. 
Abdel-Latif et al. (2020)

Table 4. Nucleotide and amino acid identities between the isolated NDV strains, reference vaccinal strains and genotype VII

1.1. strains. Genetically related strains to both lentogenic and vNDV strain are gray shaded.

\begin{tabular}{|c|c|c|c|c|c|c|c|c|c|c|c|c|c|c|c|c|c|}
\hline Virus & 1 & 2 & 3 & 4 & 5 & 6 & 7 & 8 & 9 & 10 & 11 & 12 & 13 & 14 & 15 & 16 & \\
\hline 1. II NDV/CK/MN/SA-008/17 & & 96.7 & 98.6 & 97.6 & 96.6 & 96.3 & 96.1 & 95.4 & 89.9 & 86.1 & 81.9 & 84.3 & 85.9 & 85.5 & 85.7 & 85.9 & \\
\hline 2. II NDV/CK/MN/SA-004/16 & 98.7 & & 96.7 & 96.7 & 94.2 & 96.7 & 96.7 & 95.4 & 85.4 & 85 & 81.9 & 84.3 & 85.4 & 85 & 85 & 85.4 & \\
\hline 3. II NDV/CK/FY/AM-006/18 & 99.3 & 98.6 & & 97.6 & 96.6 & 98.1 & 97.8 & 97.1 & 91.5 & 88 & 81.9 & 84.3 & 87.8 & 87.3 & 87.6 & 87.8 & \\
\hline 4. II NDV/CK/BS/AA-012/18 & 99.3 & 98.7 & 99.2 & & 96.5 & 97.6 & 97.3 & 96.5 & 90.5 & 87.8 & 81.9 & 84.3 & 88.1 & 87.5 & 87.8 & 88.1 & \\
\hline 5. II NDV/CK/BS/AM-008/18 & 98.8 & 97.6 & 98.9 & 98.6 & & 96.8 & 96.6 & 95.7 & 89.4 & 88.4 & 82.6 & 85.5 & 88.5 & 88.2 & 88.5 & 88.5 & \\
\hline 6. LaSota F gene complete & 98 & 98.7 & 99.2 & 99.2 & 98.7 & & 99.8 & 99.3 & 93.1 & 88.4 & 81.9 & 84.3 & 88.6 & 88.1 & 88.1 & 88.6 & 8 \\
\hline 7. II NDV Hitchner B1 & 97.3 & 98.2 & 98.4 & 98.6 & 98.3 & 99.2 & & 99.5 & 93 & 88.7 & 81.9 & 84.3 & 88.8 & 88.3 & 88.3 & 88.8 & : \\
\hline 8. VG/GA F gene complete & 96.9 & 97.5 & 98 & 98.2 & 97.8 & 98.9 & 99.7 & & 92.4 & 88 & 79.7 & 82.5 & 88.3 & 87.7 & 87.7 & 88.3 & \\
\hline 9. II NDV/chicken/Egypt/3/2006 & 91.1 & 87.8 & 92 & 91.6 & 91.3 & 93.6 & 93.7 & 93.4 & & 89.8 & 84.8 & 86.1 & 89.7 & 89.5 & 89.5 & 89.7 & \\
\hline 10. VII.1.1. NDV/CK/BS/AM-009/18 & 81.8 & 81.4 & 82.8 & 83.2 & 84.3 & 83.3 & 83.5 & 83.3 & 84.8 & & 98.6 & 97 & 98.9 & 98.9 & 99.1 & 98.9 & . \\
\hline 11. VII.1.1. NDV/CK/FY/SA-013/17 & 79.5 & 79.5 & 79.2 & 79.5 & 80.4 & 79.5 & 79.5 & 78.7 & 82.6 & 99 & & 96.3 & 97.1 & 97.8 & 97.8 & 97.1 & \\
\hline 12. VII.1.1. NDV/CK/FY/AA-006/18 & 81.5 & 81.5 & 81.3 & 81.5 & 82.1 & 81.5 & 81.5 & 80.9 & 84.7 & 98.8 & 98.8 & & 95.8 & 96.4 & 96.4 & 95.8 & \\
\hline 13. VII 1.1. NDV/Ck/Egypt/Dakahlia27/16 & 81.6 & 81.3 & 82.5 & 83 & 84 & 83.3 & 83.4 & 83.2 & 84.7 & 99 & 98.1 & 98 & & 99.5 & 99.5 & 100 & \\
\hline 14. VII 1.1. NDV/Ck/Egypt/Qualyobia12/16 & 81.9 & 81.7 & 82.9 & 83.4 & 84.4 & 83.4 & 83.5 & 83.3 & 84.7 & 99 & 98.1 & 98 & 99.1 & & 99.3 & 99.5 & \\
\hline 15. VII 1.1. pigeon/Eg/1095/Giza/15 & 81.9 & 81.6 & 82.9 & 83.4 & 84.3 & 83.4 & 83.5 & 83.3 & 84.8 & 98.8 & 97.8 & 97.6 & 98.8 & 99 & & 99.5 & \\
\hline \multirow[t]{2}{*}{ 16. VII 1.1. (VIId) NDV/Ck/ME13/Egypt } & 82 & 81.7 & 83 & 83.6 & 84.5 & 83.7 & 83.8 & 83.6 & 85.1 & 98.9 & 97.8 & 97.8 & 99.2 & 99 & 99.1 & & \\
\hline & \multicolumn{16}{|c|}{ Nucleotides Identity $\%$} & \\
\hline
\end{tabular}



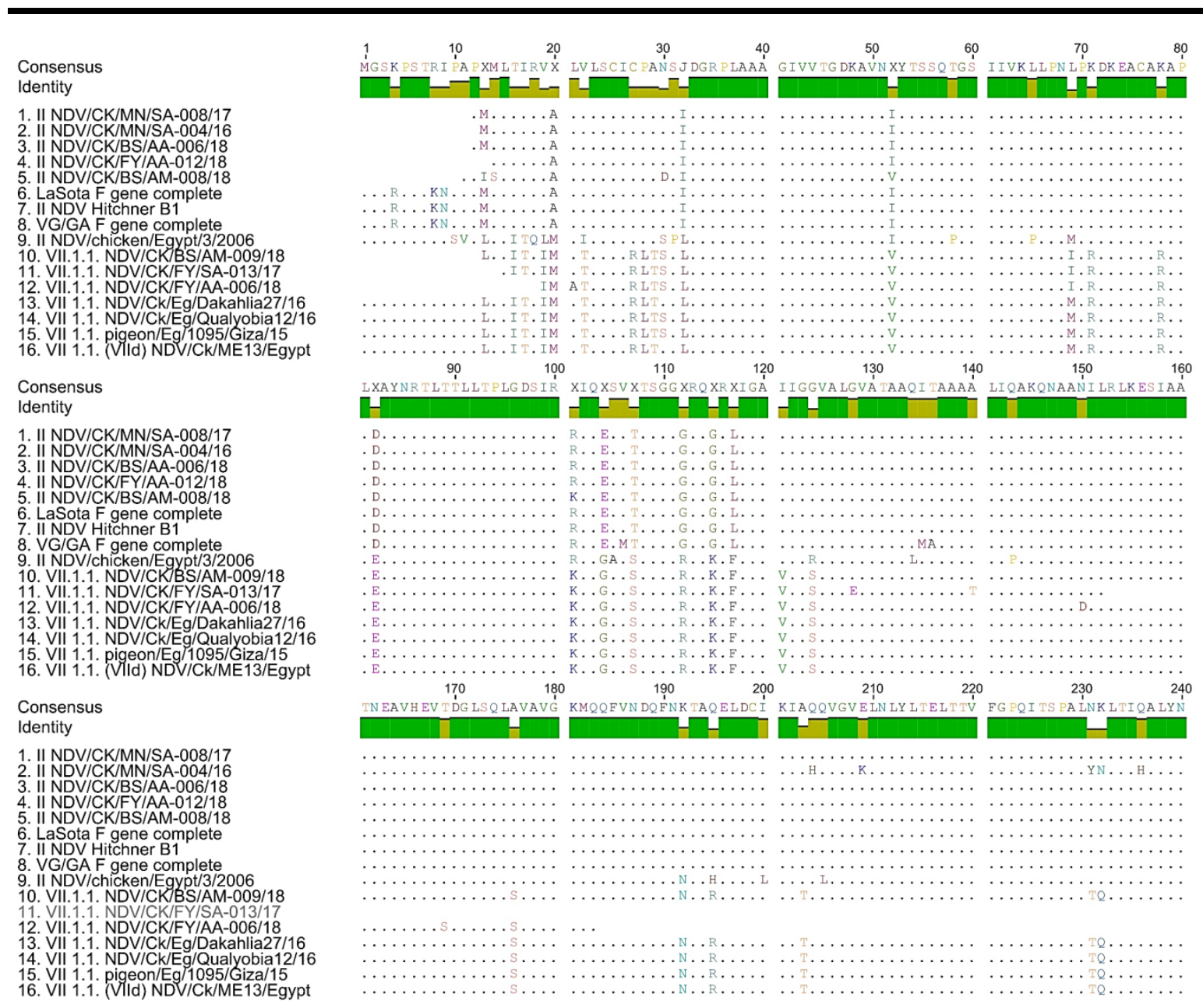

Fig. 2. Amino acid alignment of the isolated NDV strains. Grey shadow box indicate lentogenic cleavage site and red shaded box indicates velogenic cleavage site

\section{Phylogeny and Genetic Analysis of the LPAI H9N2 strains sequences}

The LPAI-H9N2 phylogeny of the HA gene showed that the 2 isolates obtained in this study are related to each other and related to recent 2016-2018 Egyptian and Middle East circulating H9N2 strains, which belonged to G1-like lineage (Figure 3). Results also showed that LPAI- H9N2 isolates share $98.4 \%$ and $98.9 \%$ nucleotide and amino acid identities in between, while they share 97.3 to $99.1 \%$ nucleotide and 97.4 to $97.7 \%$ amino acid identities with recent Egyptian strain. Notably, the 2 strains showed higher identity ( $\geq 99 \%$ ) to recent Israeli 2018 isolates (Table 5). Multiple amino acid changes were observed in the HA gene (figure 4). 


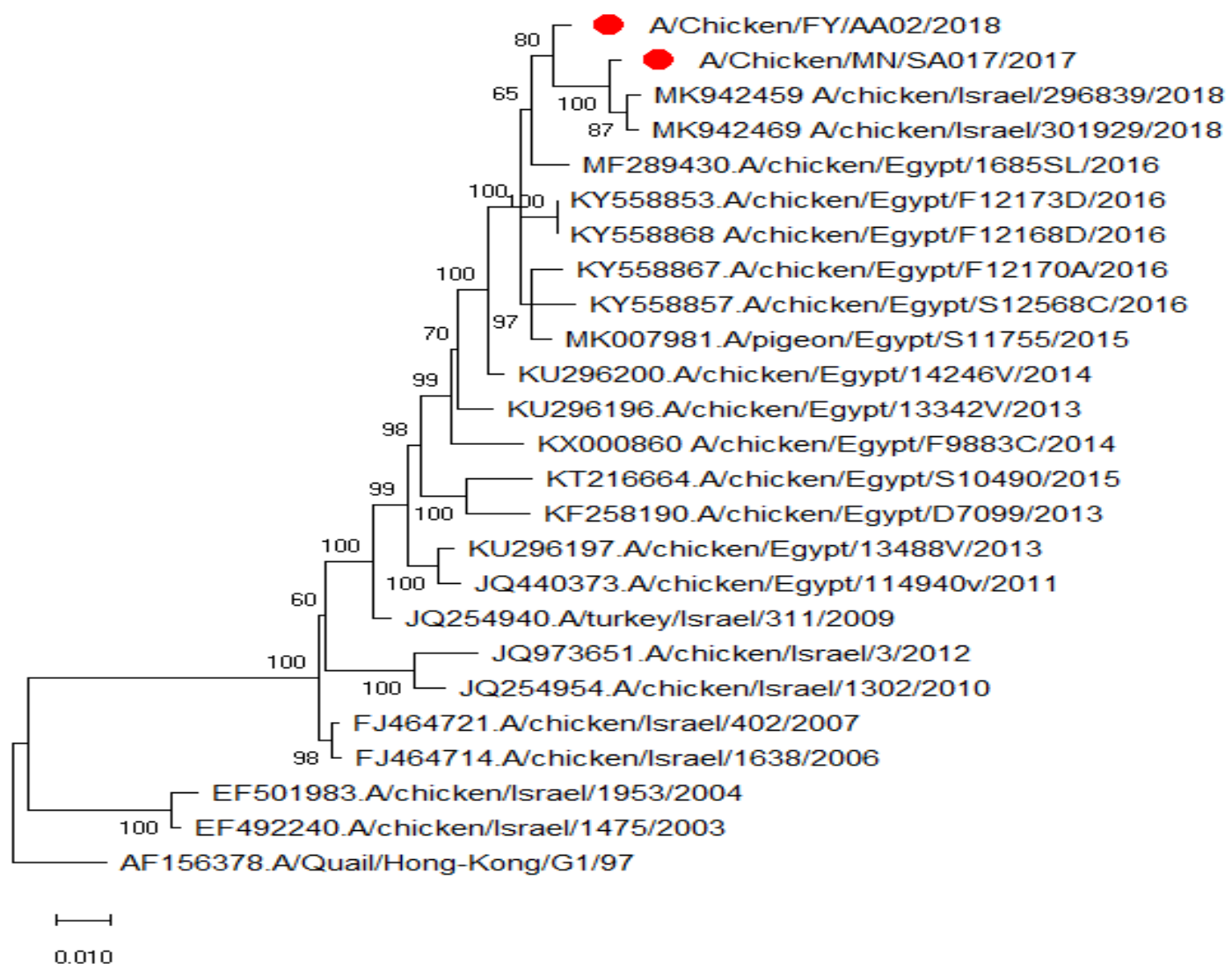

Fig. 3. Phylogenetic analysis of the HA-gene sequence of isolated LPAI-H9N2 strains (red circle •). Phylogenetic relationships through a bootstrap trial of 1000 were determined with the MEGA version 6 using the Clustal W alignment algorithm and neighbor-joining method for tree construction. 
Table 5. Nucleotide and amino acid identities between the isolated LPAI-H9N2 strains, and recent Middle East strains

\begin{tabular}{llccccccccccccccc}
\hline Virus & 1 & 2 & 3 & 4 & 5 & 6 & 7 & 8 & 9 & 10 & 11 & 12 & 13 & 14 \\
\hline 1. & A/Chicken/MN/SA017/2017 & & 98.9 & 99.4 & 99.4 & 97.9 & 97.4 & 97.4 & 95.5 & 97.7 & 97 & 96.4 & 96.4 & 95.9 & 95.1 \\
\hline 2. & A/Chicken/FY/AA002/2018 & 98.4 & & 99.1 & 98.9 & 98.7 & 98.5 & 98.5 & 96.2 & 98.6 & 98.1 & 96.8 & 96.8 & 97 & 95.6 \\
\hline 3. & MK942459 A/CK/Israel/296839/2018 & 99.3 & 98.1 & & 99.5 & 97.9 & 97.3 & 97.5 & 95.4 & 97.5 & 96.9 & 94.7 & 96.1 & 96.1 & 95 \\
\hline 4. & MK942469 A/CK/Israel/301929/2018 & 99.1 & 98 & 99.3 & & 97.9 & 97.5 & 97.7 & 95.5 & 97.5 & 96.9 & 94.7 & 96.3 & 96.1 & 95.2 \\
\hline 5. & MF289430.A/CK/Egypt/1685SL/2016 & 97.9 & 98.3 & 97.6 & 97.7 & & 98.2 & 98.4 & 96.1 & 98.8 & 98.3 & 95.9 & 96.6 & 96.6 & 95.5 \\
\hline 6. & KY558868 A/CK/Egypt/F12168D/2016 & 97.3 & 98.9 & 96.9 & 97.1 & 98.2 & & 98.8 & 96.6 & 99.2 & 98.7 & 95.6 & 97.3 & 97.3 & 96.3 \\
\hline 7. & KT216664.A/CK/Egypt/S10490/2015 & 94.6 & 95.2 & 94.1 & 94.4 & 95.5 & 95.5 & & 96.6 & 98.8 & 98.3 & 95.6 & 97.2 & 97.2 & 96.1 \\
\hline 8. & MK007981.A/PG/Egypt/S11755/2015 & 97.4 & 98.4 & 97.1 & 97.2 & 98.5 & 98.7 & 95.7 & & 96.9 & 96.9 & 97.1 & 97.1 & 97.1 & 96.6 \\
\hline 9. & KU296200.A/CK/Egypt/14246V/2014 & 97.1 & 98.1 & 96.9 & 96.9 & 98.3 & 98.5 & 96.3 & 98.5 & & 99 & 97.5 & 97.7 & 97.7 & 96.5 \\
\hline 10. KU296196.A/CK/Egypt/13342V/2013 & 96.3 & 97.1 & 95.9 & 96 & 97.4 & 97.6 & 96.4 & 97.6 & 98.5 & & 97.3 & 97.5 & 97.5 & 96.5 \\
\hline 11. KF258190.A/CK/Egypt/D7099/2013 & 95.5 & 95.9 & 93.9 & 94.1 & 95.7 & 95.2 & 97.6 & 95.3 & 96.9 & 97.2 & & 95.9 & 95.9 & 94.9 \\
\hline 12. JQ440373.A/CK/Egypt/114940v/2011 & 95.6 & 95.9 & 94.9 & 95.1 & 96 & 96.3 & 96.7 & 96.4 & 97.1 & 97.4 & 96.4 & & 97.9 & 97.2 \\
\hline 13. JQ254940.A/TK/Israel/311/2009 & 95.2 & 96.1 & 94.8 & 95 & 96 & 96.4 & 96.9 & 96.4 & 97.2 & 97.3 & 96.4 & 98.1 & 98.4 \\
\hline 14. FJ464721.A/CK/Israel/402/2007 & 94.2 & 94.8 & 93.8 & 94.1 & 95.1 & 95.3 & 95.9 & 95.3 & 96.2 & 96.2 & 95.4 & 97.3 & 98.5 & \\
\hline & & & & & & Nucleotide identity $\%$ & & & & \\
\hline
\end{tabular}


Abdel-Latif et al. (2020)
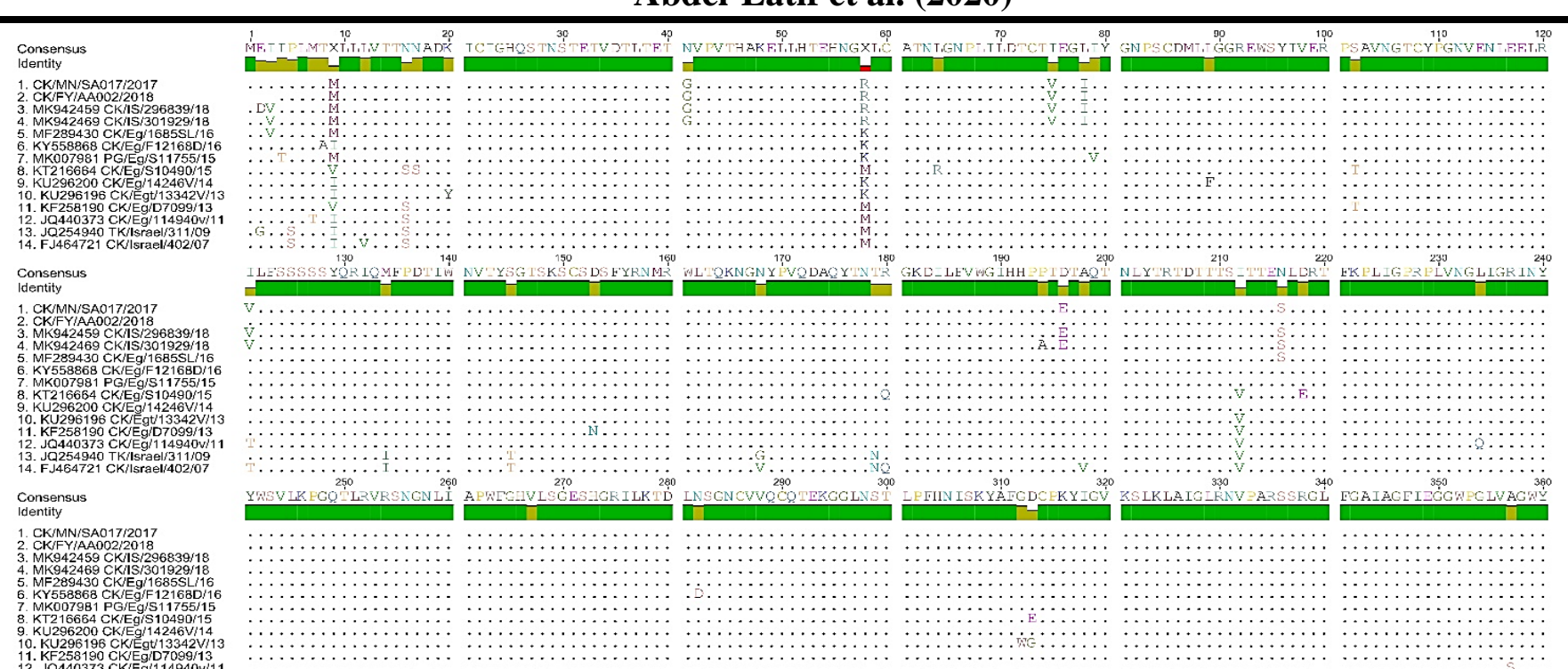

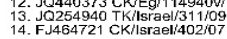

Consens

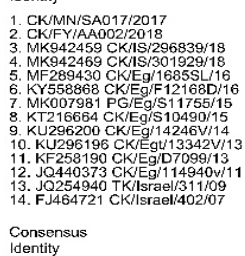

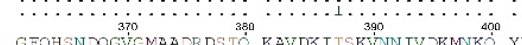

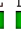

年
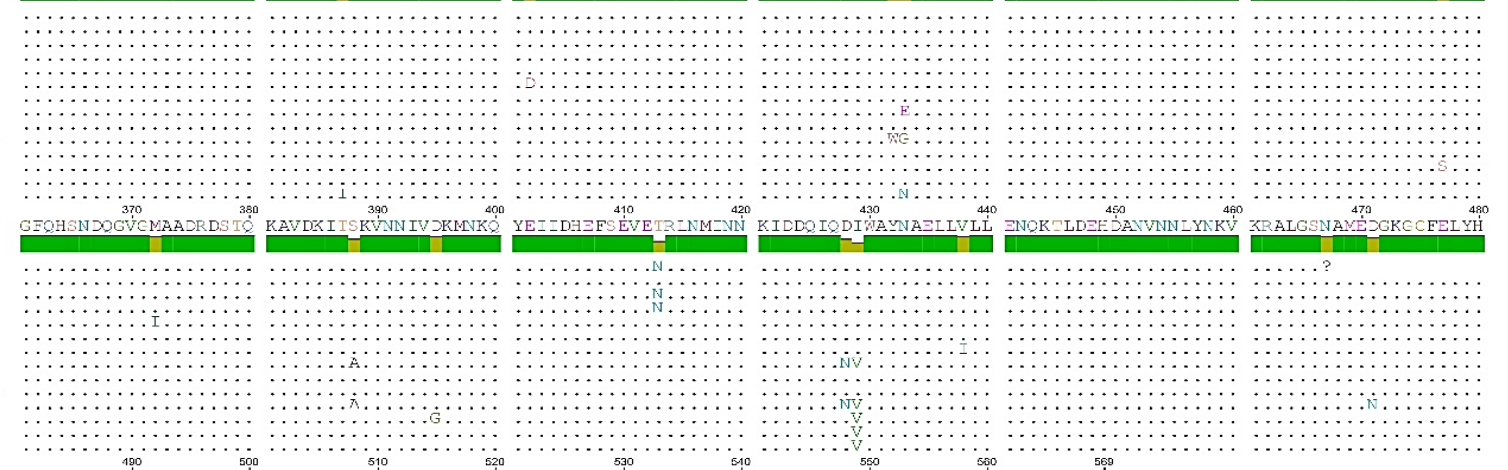

Consensus
ldentity

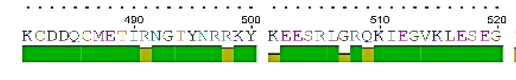

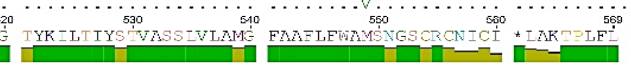
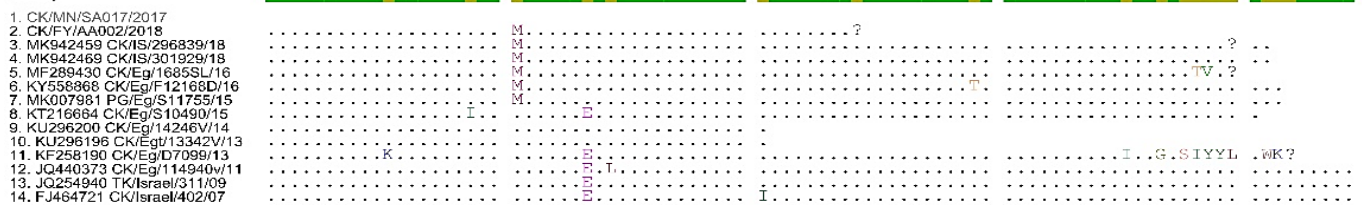

Fig. 4. Amino acid alignment of the isolated LPAI-H9N2 strains. 


\section{Discussion}

The outbreaks of LPAI-H9N2 and of NDV by genotype VII viruses usually cause severe economic losses associated with reduced performance and/or high mortalities in chicken flocks in Egypt (Ewies et al., 2017; Shehata et al., 2015). Hence, continuous surveillance is needed to monitor virus evolution under field conditions. In the current study, samples from respiratory infection outbreaks were collected from broiler chickens in Beni Suef, Fayoum, and Minia Governorates.

The postmortem examination suggested LPAI H9N2 and/or NDV viral infections and included revealed tracheitis with petechial hemorrhage on proventriculus. Virus detection using RT-PCR test revealed 37.5-46.1\% and 0$7.7 \%$ prevalence for NDV and LPAI H9N2 viruses, respectively. Both viruses are widely detected in Egyptian poultry since early 2011 (Radwan et al., 2013; Hassan et al., 2016; Orabi et al., 2017). No significant seasonal variation was observed with minimal elevation of the outbreaks in the winter season.

The analysis of selected strains $\mathrm{F}$ gene revealed the isolation of both lentogenic NDV strain and genotype VII 1.1. NDV. (Figure 1). Both genotypes retained the previously characterized F0 protein proteolytic cleavage site motifs at residues 112 to 117 (Orabi et al., 2017) with high homology to their counterpart previously identified isolates. Though NDV conventional vaccines demonstrated good efficacy to prevent clinical disease but the vaccines are unable to reduce the virus replication and shedding of currently circulating divergent virulent NDV isolates (Bello et al., 2018; Kilany et al., 2015).

This may explain the high prevalence observed in the current study despite intensive vaccination programs using various live attenuated and inactivated NDV vaccines. The results also highlight the importance of using genotype-matched vaccines to reduce the economic losses, especially under multiple viral diseases co-circulation in Egypt (Ali et al., 2019a; Ali et al., 2019b). Additionally, vaccine efficacy problems associated with the mass administration procedures, the difficulty of cold chain maintaining of the vaccines in Egypt, as well as bacterial viral co-infections due to suboptimal biosecurity practices may hinder the efficacy of the vaccines (Ali et al., 2019c; Dimitrov et al., 2017).

On the other hand, the LPAI-H9N2 isolates obtained in this study are related to each other, and related to recent 2016-2018 Egyptian and The Middle East circulating H9N2 strains, belonging to G1-like lineage. Based on nucleotide and amino acid identities, the isolates are divergent by about 5\% from the earlier isolates of 2011-2013. Recent studies showed that the Egyptian H9N2 viruses from different avian species showed several genetic markers that enhance virulence in poultry and transmission to humans and confirming that LPAI H9N2 viruses in Egypt are continuously evolving (Kandeil et al., 2017). The risk of reassortment between HPAI H5N1 and LPAI H9N2 circulating in Egypt was previously anticipated (Naguib et al., 2017). However, such event was reported with HPAI H5N8 in 2020 after their introduction to Egypt (Hagag et al., 2019; Hassan et al., 2020).

The reassortant was HPAI H5N2 virus from a commercial duck farm with the exchange of the neuraminidase segment from LPAI H9N2 (Hagag et al., 2019). Another reassortant HPAI H5N2 was detected that acquired multiple genes segments from LPAI-H9N2 isolated from both chicken and pigeon in Egypt with the HA from the HPAI H5N8 virus clade (Hassan et al., 2020). These reassortments were rather expected considering the widespread of LPAI H9N2 viruses in poultry flocks. Concurrently, the HA gene of the isolated LPAI H9N2 viruses showed multiple amino acid substitutions. These mutations are resulting from wide field circulation of the virus and/or continuous 
vaccine pressure due to the use of inactivated vaccines. Passaging of LPAI H9N2 viruses in embryonated chicken eggs with maternally derived antibodies induced selective pressure of the virus leading to genetic and antigenic variation (Jin et al., 2018) that may lead to mismatching LPAI H9N2 to the vaccine strains and even may generate pandemic strains of zoonotic risk (Meng et al., 2016).

\section{Acknowledgments}

The authors would like to thank D. Salama Shany, Assistant Professor of Poultry Diseases' Faculty of Veterinary Medicine, Beni Suef University for helping during the study.

\section{Conflict of interest}

The authors declare no conflict of interest

\section{References}

Abdelwhab, E.M., Abdel-Moneim, A.S., (2015). Epidemiology, ecology and gene pool of influenza a virus in egypt: Will egypt be the epicenter of the next influenza pandemic? Virulence 6, 6-18. 10.4161/21505594.2014.992662

Afifi, M.A.A., El-Kady, M.F., Zoelfakar, S.A., Abddel-Moneim, A.S., (2012). Serological surveillance reveals widespread influenza A $\mathrm{H} 7$ and $\mathrm{H} 9$ subtypes among chicken flocks in Egypt. Tropical animal health and production 45, 687-690.10.1007/s11250012-0243-9

Afonso, C.L., Miller, P.J., Grund, C., Koch, G., Peeters, B., Selleck, P.W., Srinivas, G.B. (2012). Newcastle disease virus (infection with Newcastle disease virus) In The OIE Manual of Diagnostic Tests and Vaccines for Terrestrial Animals. (OIE).

Ali, A., I. Abd El-Mawgoud, A., M. Dahshan, A.-H., A. EL-Sawah, A., A. Nasef, S., (2019a). Escherichia coli in broiler chickens in Egypt, its virulence traits and vaccination as an intervention strategy. Novel Research in Microbiology Journal 3, 415-427. 10.21608/nrmj.2019.44950
Ali, A., Safwat, M., Kilany, W.H., Nagy, A., Shehata, A.A., Zain El-Abideen, M.A., Dahshan, A.-H.M., Arafa, A.-S.A., (2019b). Combined h5nd inactivated vaccine protects chickens against challenge by different clades of highly pathogenic avian influenza viruses subtype h5 and virulent newcastle disease virus. Veterinary world 12, 97105. 10.14202/vetworld.2019.97-105

Ali, A.M., Samy, M.M., Fasina, F.O., Hassan, M.K., Kilany, W.H., El-Mahdy, S., Saad, A., Lubroth, J., Jobre, Y., (2019c). Field evaluation of common poultry viral vaccinesin egypt: A need for reassessment of the vaccine value chain. Veterinaria italiana 55, 231-239. 10.12834/VetIt.999.5287.1

Bello, M.B., Yusoff, K., Ideris, A., Hair-Bejo, M., Peeters, B.P.H., Omar, A.R., (2018). Diagnostic and vaccination approaches for newcastle disease virus in poultry: The current and emerging perspectives. Biomed Res Int 2018, 7278459. 10.1155/2018/7278459

Czeglédi, A., Ujvári, D., Somogyi, E., Wehmann, E., Werner, O., Lomniczi, B., (2006). Third genome size category of avian paramyxovirus serotype 1 (newcastle disease virus) and evolutionary implications. Virus Research $\quad 120, \quad 36-48$. 10.1016/j.virusres.2005.11.009

Dimitrov, K.M., Abolnik, C., Afonso, C.L., Albina, E., Bahl, J., Berg, M., Briand, F.X., Brown, I.H., Choi, K.S., Chvala, I., Diel, D.G., Durr, P.A., Ferreira, H.L., Fusaro, A., Gil, P., Goujgoulova, G.V., Grund, C., Hicks, J.T., Joannis, T.M., Torchetti, M.K., Kolosov, S., Lambrecht, B., Lewis, N.S., Liu, H., Liu, H., McCullough, S., Miller, P.J., Monne, I., Muller, C.P., Munir, M., Reischak, D., Sabra, M., Samal, S.K., Servan de Almeida, R., Shittu, I., Snoeck, C.J., Suarez, D.L., Van Borm, S., Wang, Z., Wong, F.Y.K., (2019). Updated unified phylogenetic classification system and revised nomenclature for Newcastle disease virus. 
Infection, genetics and evolution : journal of molecular epidemiology and evolutionary genetics in infectious diseases 74, 103917.10.1016/j.meegid.2019.103917

Dimitrov, K.M., Afonso, C.L., Yu, Q., Miller, P.J., (2017). Newcastle disease vaccinesa solved problem or a continuous challenge? Veterinary microbiology 206, 126-136. 10.1016/j.vetmic.2016.12.019

El-Zoghby, E.F., Arafa, A.S., Hassan, M.K., Aly, M.M., Selim, A., Kilany, W.H., Selim, U., Nasef, S., Aggor, M.G., Abdelwhab, E.M., Hafez, H.M., (2012). Isolation of $\mathrm{h} 9 \mathrm{n} 2$ avian influenza virus from bobwhite quail (colinus virginianus) in egypt. Archives of Virology 157, 1167-1172. 10.1007/s00705-012-1269-z

Ewies, S.S., Ali, A., Tamam, S.M., Madbouly, H.M., (2017). Molecular characterization of newcastle disease virus (genotype vii) from broiler chickens in egypt. BeniSuef University Journal of Basic and Applied Sciences 6, 232-237. 10.1016/j.bjbas.2017.04.004

Fouchier, R.A., Bestebroer, T.M., Herfst, S., Van Der Kemp, L., Rimmelzwaan, G.F., Osterhaus, A.D., (2000). Detection of influenza a viruses from different species by pcr amplification of conserved sequences in the matrix gene. Journal of clinical microbiology 38, 4096-4101.

Hagag, N.M., Erfan, A.M., El-Husseiny, M., Shalaby, A.G., Saif, M.A., Tawakol, M.M., Nour, A.A., Selim, A.A., Arafa, A.S., Hassan, M.K., Hassan, W.M.M., Fahmy, H.A., Ibraheem, E., Attia, M., Abdelhakim, A.M.M., Shahein, M.A., Naguib, M.M., (2019). Isolation of a novel reassortant highly pathogenic avian influenza (h5n2) virus in egypt. Viruses 11. 10.3390/v11060565

Hassan, K.E., Ali, A., Shany, S.A.S., El-Kady, M.F., (2017). Experimental co-infection of infectious bronchitis and low pathogenic avian influenza h9n2 viruses in commercial broiler chickens.
Research in veterinary science $115,356-$ 362. 10.1016/j.rvsc.2017.06.024

Hassan, K.E., King, J., El-Kady, M., Afifi, M., Abozeid, H.H., Pohlmann, A., Beer, M., Harder, T., (2020). Novel reassortant highly pathogenic avian influenza $\mathrm{a}(\mathrm{h} 5 \mathrm{n} 2)$ virus in broiler chickens, egypt. Emerg Infect Dis 26, 129-133. 10.3201/eid2601.190570

Hassan, K.E., King, J., El-Kady, M., Afifi, M., Abozeid, H.H., Pohlmann, A., Beer, M., Harder, T., (2020). Novel Reassortant Highly Pathogenic Avian Influenza A(H5N2) Virus in Broiler Chickens, Egypt. Emerg Infect Dis 26, 129133.10.3201/eid2601.190570

Hassan, K.E., Shany, S.A.S., Ali, A., Dahshan, A.H.M., El-Sawah, A.A., El-Kady, M.F., 2016. Prevalence of avian respiratory viruses in broiler flocks in egypt. Poultry science 95, 1271-1280. 10.3382/ps/pew068

Hoffmann, E., Stech, J., Guan, Y., Webster, R.G., Perez, D.R., (2001). Universal primer set for the full-length amplification of all influenza a viruses. Archives of Virology 146, 2275-2289. 10.1007/s007050170002

Jin, H., Wang, W., Yang, X., Su, H., Fan, J., Zhu, R., Wang, S., Shi, H., Liu, X., (2018). Evolution of h9n2 avian influenza virus in embryonated chicken eggs with or without homologous vaccine antibodies. BMC veterinary research 14, 71. 10.1186/s12917-0181391-6

Kandeil, A., El-Shesheny, R., Maatouq, A., Moatasim, Y., Cai, Z., McKenzie, P., Webby, R., Kayali, G., Ali, M.A., (2017). Novel reassortant h9n2 viruses in pigeons and evidence for antigenic diversity of $\mathrm{h} 9 \mathrm{n} 2$ viruses isolated from quails in egypt. The Journal of general virology $\quad 98, \quad 548-562$. 10.1099/jgv.0.000657 
Karimi-Madab, M., Ansari-Lari, M., Asasi, K., Nili, H., (2010). Risk factors for detection of bronchial casts, most frequently seen in endemic h9n2 avian influenza infection, in poultry flocks in iran. Preventive Veterinary Medicine 95, 275-280.

10.1016/j.prevetmed.2010.03.010

Kilany, W.H., Ali, A., Bazid, A.H.I., Zain ElAbideen, M.A., El Sayed, M., (2015). Evaluation of two inactivated newcastle disease virus vaccines (genotype ii and vii) against challenge of newcastle disease genotype vii infection in chicken. Journal of Animal and Veterinary Advances 14, 211-218. 10.3923/javaa.2015.211.218

Kumar, S., Stecher, G., Li, M., Knyaz, C., Tamura, K., (2018). Mega x: Molecular evolutionary genetics analysis across computing platforms. Mol Biol Evol 35, 1547-1549. 10.1093/molbev/msy096

Meng, F., Xu, H., Zhang, W., Huang, D., Zhang, Z., Liu, X., Chang, W., Qin, Z., (2016). Genetic evolution and substitution frequency of avian influenza virus ha gene in chicken h9n2 subtype in china in the last 20 years. Wei sheng wu xue bao = Acta microbiologica Sinica 56, 35-43.

Miller, P.J., E. L. Decanini, and C. L. Afonso., (2010). Newcastle disease: Evolution of genotypes and the related diagnostic challenges. Infect. Genet. Evol. .

Mohamed, M.H., Kumar, S., Paldurai, A., Samal, S.K., (2011). Sequence analysis of fusion protein gene of newcastle disease virus isolated from outbreaks in egypt during 2006. Virology journal 8, 237. 10.1186/1743-422X-8-237

Naguib, M.M., Arafa, A.-S., Parvin, R., Beer, M., Vahlenkamp, T., Harder, T.C., (2017). Insights into genetic diversity and biological propensities of potentially zoonotic avian influenza h9n2 viruses circulating in egypt. Virology 511, 165174. 10.1016/j.virol.2017.08.028
Nagy, A., Ali, A., Zain El-Abideen, M.A., Kilany, W., Elsayed, M., (2020). Characterization and genetic analysis of recent and emergent virulent newcastle disease viruses in Egypt. Transboundary and diseases.10.1111/tbed.13543 emerging

Nagy, A., Mettenleiter, T.C., Abdelwahab, E.M., (2017). A brief summary of the epidemiology and genetic relatedness of avian influenza $h 9 n 2$ virus in birds and mammals in the middle east and north africa. Epidemiology and infection 145, 3320-3333. $10.1017 / \mathrm{S} 0950268817002576$

Nili, H., Asasi, K., (2002). Natural cases and an experimental study of $\mathrm{h} 9 \mathrm{n} 2$ avian influenza in commercial broiler chickens of iran. Avian Pathology 31, 247-252. 10.1080/03079450220136567

Orabi, A., Hussein, A., Saleh, A.A., El-Magd, M.A., Munir, M., (2017). Evolutionary insights into the fusion protein of newcastle disease virus isolated from vaccinated chickens in 2016 in egypt. Archives of Virology 162, 3069-3079. 10.1007/s00705-017-3483-1

Peyre, M., Samaha, H., Makonnen, Y.J., Saad, A., Abd-Elnabi, A., Galal, S., Ettel, T., Dauphin, G., Lubroth, J., Roger, F., Domenech, J., (2009). Avian influenza vaccination in egypt: Limitations of the current strategy. Journal of molecular and genetic medicine : an international journal of biomedical research 3, 198204.

Radwan, M.M., Darwish, S.F., El-Sabagh, I.M., El-Sanousi, A.A., Shalaby, M.A., (2013). Isolation and molecular characterization of newcastle disease virus genotypes ii and viid in egypt between 2011 and 2012. Virus Genes 47, 311-316. 10.1007/s11262-013-0950-y

Samy, A., Naguib, M.M., (2018). Avian respiratory coinfection and impact on avian influenza pathogenicity in 
domestic poultry: Field and experimental findings. Vet Sci 5 . $10.3390 /$ vetsci5010023

Setta, A., Salem, H.M., Elhady, M., ElHussieny, A., Arafa, A.S., (2018). Molecular and genetic characterization of infectious bronchitis viruses isolated from commercial chicken flocks in egypt between 2014 and 2016. Journal of World's Poultry Research 8, 1-7.

Shany, S.A.S., (2015). Further studies on the current situation of avian influenza in egypt, $\mathrm{PhD}$. Thesis, Poultry Diseases Department, Beni-Suef University, Egypt.
Shehata, A.A., Parvin, R., Sultan, H., Halami, M.Y., Talaat, S., Abd Elrazek, A., Ibrahim, M., Heenemann, K., Vahlenkamp, T., (2015). Isolation and full genome characterization of avian influenza subtype h9n2 from poultry respiratory disease outbreak in egypt. Virus Genes 50, 389-400. 10.1007/s11262-015-1188-7

Swayne, D., Brown, I. (2015). Chapter 3.3.4. Infection with avian influenza viruses, In: Manual of diagnostic tests and vaccines for terrestrial animals. OIE. 